\title{
Realizing Two-dimensional Spin Arrays on Surfaces via Halogen-bonding Molecular Self-assembly
}

Dingguan Wang,,${ }^{[\mathrm{a}]+}$ Zishen Wang, ${ }^{[\mathrm{a}]+}$ Shaofei Wu, ${ }^{[\mathrm{b}]+}$ Arramel, ${ }^{[\mathrm{a}]}$ Xinmao Yin, ${ }^{[\mathrm{c}][\mathrm{e}]}$ Chi Sin Tang, ${ }^{[\mathrm{d}][\mathrm{e}]}$ Yuan Ping Feng, ${ }^{[\mathrm{a}]}$ Jishan $\mathrm{Wu}^{*[\mathrm{~b}]}$ and Andrew Thye Shen Wee*[a]

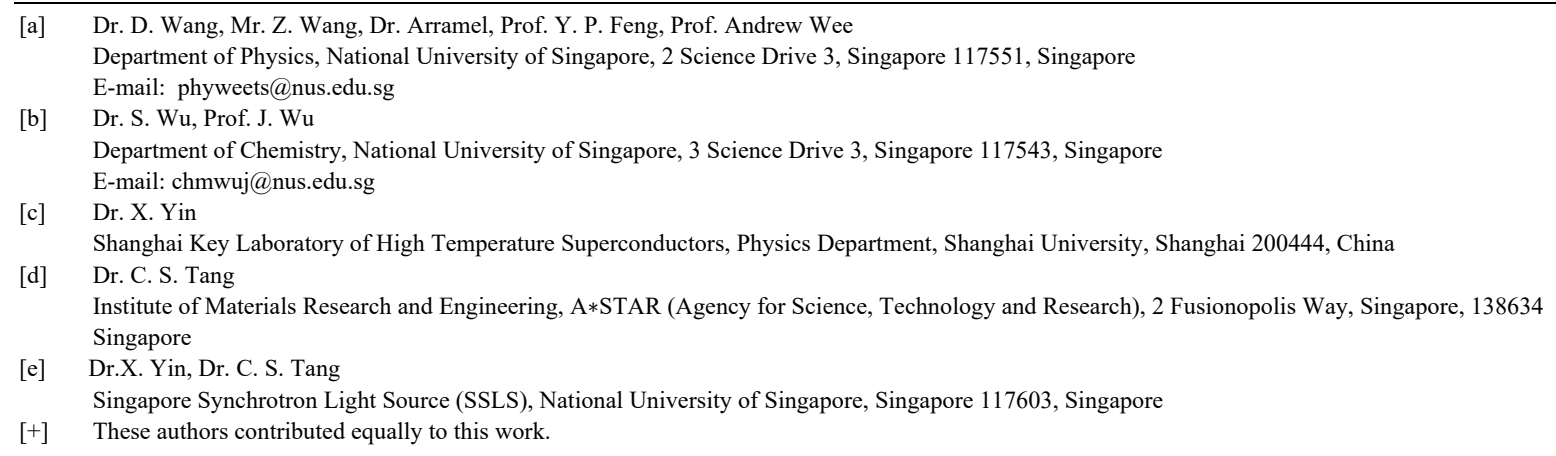

Keywords: organic radicals, 2D spin arrays, halogen bonds, scanning tunneling microscopy, DFT calculations

\begin{abstract}
Well-ordered spin arrays are highly desirable for next-generation molecule-based magnetic devices, and yet its synthetic method remains a challenging task. Herein, we demonstrate the realization of two-dimensional supramolecular spin arrays on surfaces via halogen-bonding molecular self-assembly. A bromine-terminal perchlorotriphenymethyl radical with net carbon spin was synthesized and deposited on $\mathrm{Au}(111)$ to achieve twodimensional supramolecular spin arrays. By taking advantage of the diversity of halogen bonds, five supramolecular spin arrays are presented with ultrahigh spin densities (up to the value of $3 \times 10^{13}$ spins at the size of a flash drive), as probed by low-temperature scanning tunneling microscopy at the single-molecule level. First principle calculations verify that the formation of three distinct types of halogen bonds can be used to tailor supramolecular phases via molecular coverage and annealing temperature. Our work demonstrates supramolecular self-assembly as a promising method to engineering $2 \mathrm{D}$ spin arrays for potential application in magnetic devices.
\end{abstract}




\section{Introduction}

Single-molecule magnets (SMMs) are a distinct magnetic entity composed of a single molecule which was built using bottom-up approach at the nanoscale. SMMs have attracted much interest due to their potential applications in ultra-high density information storage, ${ }^{1,2}$ quantum computers, ${ }^{3,4}$ spintronic devices. ${ }^{5,6}$ In 1993, Sessoli et al. reported that a SMM of metal-organic compound can be realized with high spin states. ${ }^{7}$ Metal-organic compounds consists of transition metals and organic-ligands are the research focus of SMMs, and various type of metal-organic magnets have been reported. ${ }^{8-12}$ Unlike metal spins which origin from unpaired electron in $d$ - or $f$-orbital, organic carbon is another spin element that generates its magnetism due to an unpaired electron in $p$-orbital. Recent studies have shown that through molecular design, organic molecules could exhibit an open-shell ground state, revealing emergent SMMs with a net carbon spin. ${ }^{13-17}$

The successful synthesis of high-quality SMMs enables to further research on their device applications. Fabricating ordered spin arrays of SMMs is an important step towards practical implementations. A method so called crystal engineering has been introduced to control over the ordered molecular architectures and align the spin orientation/distance of SMMs into one-, two- or three-dimensional spin arrays, such that spin exchange interaction and the resulting magnetic properties can be tuned efficiently. ${ }^{15,18,19}$ In particular, the realization of twodimensional spin arrays on a substrate has attracted much interest, since it is a key step to the applications in high-density storage devices and spintronic devices. ${ }^{20}$ Until now, several reports have demonstrated two-dimensional molecular spins via the deposition of $\mathrm{SMMs}^{21,22}$ or on-surface synthesis of molecular magnets on a substrate. ${ }^{23-28}$ However, due to the lack of an effective means to regulate the molecular organization of these magnets, it is still a great challenge to achieve a highly-ordered two-dimensional spin arrays on surface. ${ }^{29,} 30$ 
Two-dimensional molecular self-assembly has shown great advantages in achieving supramolecular arrays on surfaces. ${ }^{31-35}$ By means of intermolecular interactions including hydrogen bonds, dipole-dipole interactions, coordination bonds, van der Waals forces (vdW), halogen bonds, many ordered supramolecular patterns have been reported, ranging from zerodimensional, one-dimensional to two-dimensional supramolecular arrays. ${ }^{36-42}$ In particular, by employing high-resolution surface characterization techniques, halogen bonds have recently been studied and show great promise in mediating highly-ordered supramolecular arrays on surface. ${ }^{43-46}$

In this work, a bromine-terminated perchlorotriphenymethyl radical (3Br-PTM) with net carbon spin was synthesized and thermally-deposited to achieve two-dimensional ordered spin arrays on an insulating bromine monolayer on $\mathrm{Au}(111)$. Halogen bonds play an important role in regulating the supramolecular spin arrays formation on surface. By taking advantage of the diversity of halogen bonds, five ordered supramolecular architectures with variable spin densities and distances were observed using high-resolution LT-STM. DFT calculations further show that the observed spin arrays are mainly governed from three distinct types of halogen bonds, namely, linear single Br-bond (Type-1 halogen bond), triangular triple Br-bonds (Type-2 halogen bond) and hexagonal sextuple Br-bonds (Type-3 halogen bond).

\section{Results and Discussion}

3Br-PTM molecule was synthesized via solution-based organic synthesis (Figure S1 in the Supporting Information). Figure 1a displays a 3D molecular structure of 3Br-PTM that was obtained from the single crystal XRD measurement (CCDC 2088008). The 3Br-PTM backbone is a triangular-shaped molecule with $D_{3}$-symmetry. The $3 \mathrm{Br}-\mathrm{PTM}$ is considered a nonplanar molecule since two adjacent benzene rings are rotated with respect to the plane of the molecule due to steric hindrance. Since the 3Br-PTM molecule has an odd number of $s p^{2}$ 
carbons (37 carbons), a single electron in $p$-orbital of the central carbon does not participate in the pairing $\pi$-bonds, which leads to the formation of carbon-centered net spin. ${ }^{47}$ In order to verify our hypothesis, electron spin resonance (ESR) measurements were carried out. As shown in Figure 1b, an intense one-line ESR signal is observed with the $g$ value of 2.0029, verifying a typical carbon-centered spin. DFT calculations also support our expected hypothesis and the ESR observation. Figure 1c shows the calculated spin density distribution of a $3 \mathrm{Br}-\mathrm{PTM}$ molecule, which shows that the spin is mainly localized at the central carbon atom. Moreover, the spin intensity of $1.0 \mu \mathrm{B}$ per molecule in gas phase is obtained according to DFT calculation in line to the typical net-spin molecule.

a

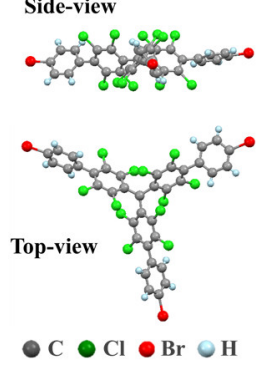

C

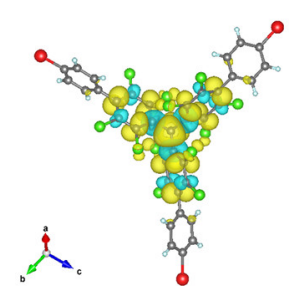

b

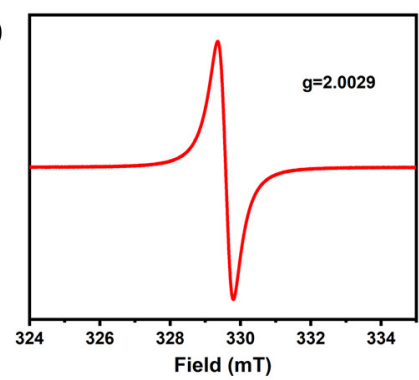

d

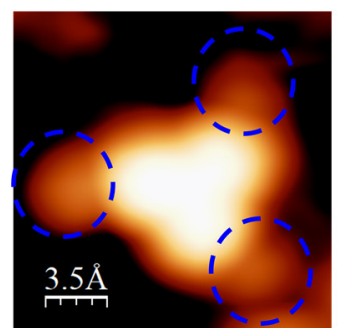

Figure 1. Molecular spin originated from an unpaired electron in the central carbon of $3 \mathrm{Br}$ PTM molecule. (a) Chemical structure of 3Br-PTM molecule, the side-view and top-view of 3Br-PTM molecule are obtained from the single crystal XRD. (b) Solid-state ESR spectrum of $3 \mathrm{Br}-\mathrm{PTM}$ powder at $293 \mathrm{~K}$, and the $g$ value of 2.0029 indicates a typical carbon-centred spin. (c) Calculated spin density distribution of 3Br-PTM molecule, yellow and light blue isosurfaces indicate spin up and spin down, respectively. (d) High-resolution STM image of a single 3Br-PTM molecule on $\mathrm{Au}(111)$, three outer Br-terminated benzene rings are labeled by blue circles, set point: $V_{\text {tip }}=-0.2 \mathrm{~V}$ and $I_{\text {tip }}=38 \mathrm{pA}$.

The 3Br-PTM molecules were thermally deposited on $\mathrm{Au}(111)$ substrate under ultra-high vacuum, and the as-grown samples were subsequently in-situ transferred into LT-STM 
chamber for imaging at $77 \mathrm{~K}$. Figure 1d exhibits a high-resolution STM image of a single 3Br-PTM molecule on $\mathrm{Au}(111)$. After thermal deposition, the 3Br-PTM molecule prefers to lie flat on surface. The molecule presents a triangular geometry with $D_{3}$-symmetry, consistent with our observation in the single crystal XRD determination in Figure 1a. We noted that due to the twisted feature of the adjacent benzene rings (the outer and inner benzene rings), their contrast variation shown in the STM image reflects that three outer bright spots are assigned to the outer Br-terminated benzene rings (labeled by blue circles), while the other three brighter spots (clover-like structure) are associated to the three inner benzene rings.

Figure 2 presents the vertically-stacked heterostructures consisting of 3Br-PTM submonolayer on Br-atom monolayer on $\mathrm{Au}(111)$ substrate. Figure 2a shows a low molecular coverage STM image obtained via depositing 3Br-PTM molecule on $\mathrm{Au}(111)$ at $180{ }^{\circ} \mathrm{C}$ for 3 minutes. Two distinct domains are observed, one domain is covered by $\mathrm{Br}$ atoms while another is covered by ordered molecules, as shown in Figure $2 \mathrm{~b}$ and $2 \mathrm{c}$, respectively. Figure $2 \mathrm{~b}$ shows a zoom-in STM image of the Br atom monolayer. The bottom layer consists of close-packed hexagonal bright spots (labeled by a blue arrow in Figure 2b), and its measured unit cell is $0.53 \pm 0.02 \times 0.53 \pm 0.02 \mathrm{~nm}$. It can be assumed that the formation of a $\mathrm{Br}$ monolayer is induced by the partial cleavage of $\mathrm{Br}$ atoms from the 3Br-PTM molecules upon thermal evaporation onto the $\mathrm{Au}(111)$ substrate. A few of molecules (labeled by blue arrows) adsorb on the top of the Br-monolayer. DFT calculations in Figure 2d highlights the Br atoms preferably absorb on $h c p$ sites of $\mathrm{Au}(111)$ substrate to form a close-packed hexagonal structure. The calculated unit cell is $0.54 \mathrm{~nm} \times 0.54 \mathrm{~nm}$, which is double of $\mathrm{Au}(111)$ substrate lattice $(0.27 \mathrm{~nm})$, and this result is comparable to the extracted lattice periodicity $(\sim 0.53 \mathrm{~nm})$ from STM image. 


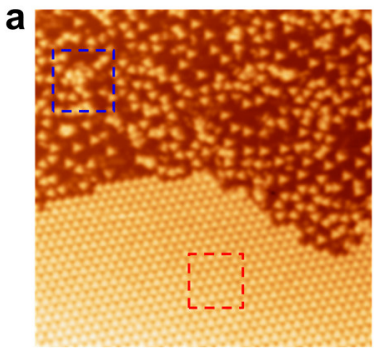

d

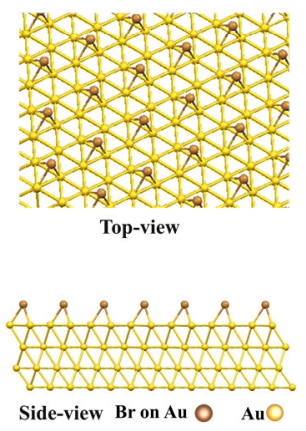

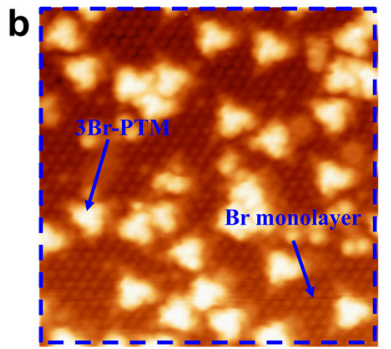

e

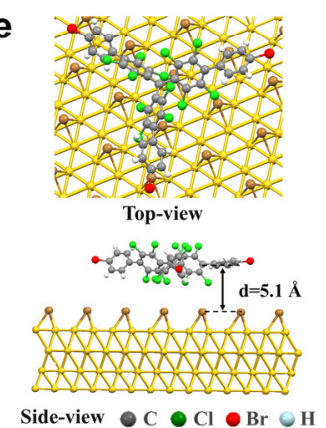

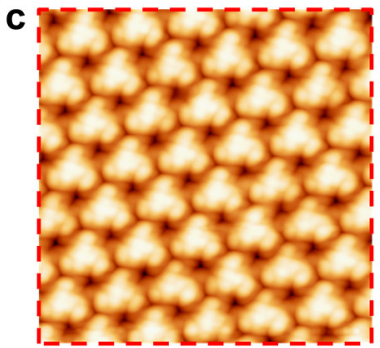

f

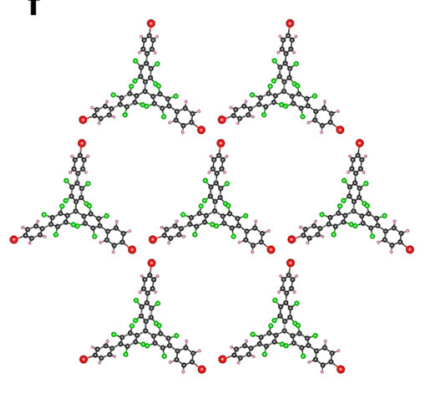

Figure 2. 3Br-PTM sub-monolayer/Br-monolayer/Au(111). (a) STM image of 3Br-PTM submonolayer on $\mathrm{Br}$ monolayer/ $\mathrm{Au}(111)$ after depositing at $180{ }^{\circ} \mathrm{C}$ for $3 \mathrm{mins}$, set point: $V_{t i p}=$ $1.0 \mathrm{~V}$ and $I_{\text {tip }}=30 \mathrm{pA}$, size: $60 \mathrm{~nm} \times 60 \mathrm{~nm}$. (b) Zoom-in STM image of a few 3Br-PTM molecules on Br-monolayer on $\mathrm{Au}(111)$, set point: $V_{\text {tip }}=1.0 \mathrm{~V}$ and $I_{\text {tip }}=30 \mathrm{pA}$, size: $15 \mathrm{~nm} \times$ $15 \mathrm{~nm}$. (c) Zoom-in STM image of phase-1 domain; set point: $V_{\text {tip }}=0.8 \mathrm{~V}$ and $I_{\text {tip }}=30 \mathrm{pA}$, size: $10 \mathrm{~nm} \times 10 \mathrm{~nm}$. (d) Calculated atomic structure of Br-monolayer on $\mathrm{Au}(111)$, calculated unit cell: $5.4 \mathrm{~nm} \times 5.4 \mathrm{~nm}$; The $\mathrm{Br}$ atoms absorb on $h c p$ site, by contrast, Br monolayer is energetically unstable when absorbed on $f c c$ site, as suggested by DFT calculations. (e) Calculated atomic structure of a 3Br-PTM molecule on Br-monolayer on Au(111). (f) DFToptimized molecular packing structure of phase-1 domain.

When the molecules were deposited at a lower temperature of $150^{\circ} \mathrm{C}$, we do not observe a similar 3Br-PTM molecules arrangement, and only the $\mathrm{Br}$ sub-monolayer is observed in Figure S2a. The X-ray Photoemission (XPS) measurements of the 3Br-PTM monolayer on Br-monolayer on $\mathrm{Au}(111)$ in Figure $\mathrm{S} 3$ also show two distinct $\mathrm{Br}-3 d$ peaks, which originate from two different chemical states of $\mathrm{Br}-\mathrm{C}$ and $\mathrm{Br}-\mathrm{Au}$ bonding. ${ }^{48}$ Figure $\mathrm{S} 2 \mathrm{~b}$ shows that the thickness of the $\mathrm{Br}$ monolayer is about $0.13 \mathrm{~nm}$. This small distance suggests the strong chemisorption of $\mathrm{Br}$ atom on $\mathrm{Au}(111)$, which is also supported by the disappearance of $\mathrm{Au}(111)$ herringbone reconstructions in Figure $2 \mathrm{a}$ and Figure S4a. ${ }^{49}, 50$ In contrast, 3Br-PTM molecules are not strongly absorbed on the Br-monolayer. The orientation of each individual 
3Br-PTM molecule is random, and the corresponding molecular thickness is higher relative to the Br-monolayer $(\sim 0.28 \mathrm{~nm}$ in Figure $\mathrm{S} 2 \mathrm{~d})$. We infer that the adsorption of 3Br-PTM molecules on $\mathrm{Br}$ monolayer is a weak physical adsorption.

To verify the hypothesis of a weak physical adsorption of 3Br-PTM molecules on $\mathrm{Br}$ monolayer, DFT calculation was performed in order to resolve the estimated molecular binding energy. Figure 2e shows that the interlayer distance between the 3Br-PTM molecule and $\mathrm{Br}$ monolayer is very large $(0.51 \mathrm{~nm})$, which is beyond the range of chemisorption, suggesting that the molecular adsorption of the 3Br-PTM molecule on Br-monolayer is considered as physisorption. On the other hand, DFT calculation shows that the molecular binding energy is only $\sim 81 \mathrm{meV}$ per atom, and the absorption energy was reduced by $1 \%$ upon absorption, verifying that the effect of the $\mathrm{Br}$-monolayer/Au(111) substrate on the molecule is very weak. This result is reasonable since Br-monolayer has a large energy gap and is used as an insulating buffer layer. ${ }^{51}$

The molecular magnetic property is maitained up on absorption on the buffer $\mathrm{Br}$ monolayer/Au(111). According to the calculated results, the magnetic strength of $3 \mathrm{Br}-$ $\mathrm{PTM} / \mathrm{Br}-\mathrm{monolayer} / \mathrm{Au}(111)$ is $0.24 \mu \mathrm{B}$ per molecule. This could be a result of incomplete shielding by the Au substrate due to the presence of the buffer Br-monolayer ${ }^{52}$ which reduces the charge transfer between the molecule and the substrate. ${ }^{53}$ Compared with the isolated molecule in gas phase, the shielding effect of the substrate on molecular magnetic property cannot be ignored in 3Br-PTM/Br-monolayer/Au(111). The DFT calculation in Figure S5 verifies the shielding effect from substrate, in which spin density transfers from 3Br-PTM molecule to Br-monolayer is observed.

Figure 2c displays a zoom-in STM image of 3Br-PTM domain on Br-monolayer on Au(111). In this domain, a highly-ordered molecular array is unambiguously observed, which is labeled as phase-1. We infer the formation of triple Br-halogen bonds since three Br-terminals are adjacent. The DFT-optimized molecular packing structure in Figure $2 \mathrm{f}$ supports our STM 
observation, suggesting that triple Br-halogen bonds play an important role on the selfassembly of the 3Br-PTM molecules into highly-ordered carbon spin arrays, as further discussed in Figure 4.

Figure 3 presents the multi-phases of 3Br-PTM self-assembled on Br-monolayer on $\mathrm{Au}(111)$ at high molecular coverage. Five molecular self-assembled phases were observed when the molecular coverage is high ( $\sim 0.9 \mathrm{ML})$, which can be achieved by increasing the deposition time. Figure 3a represents phase-2 with highly-packed and ordered structure at a coverage of $\sim 0.9$ ML. We observe the existence of multi-phases, and Figure S6 gives a large-scale STM image of the mixed phase-1 \& -2 on Br-monolayer on Au(111). The high-resolution STM image in Figure $3 \mathrm{~b}$ shows that six 3Br-PTM molecules form a close-packed ring-like architecture with $\mathrm{X}_{6}$-synthon structure. Figure $3 \mathrm{c}$ presents a DFT-optimized molecular packing structure of phase-2, which reproduces the STM observation. The optimized structure suggests that six bromine atoms form a hexagon, and each of these bromine atoms interacts with two neighboring bromine atoms through $\mathrm{Br}-$ halogen bond. We infer that the formation of hexagonal sextuple Br-bonds (Type-3 halogen bonds) promotes 3Br-PTM molecular assembly into phase-2 spin arrays, as elaborated in Figure 4.

The emergence of phase-3 is commonly observed in other areas, with its peculiar hexagonal porous structures. Figure 3d exhibits high-resolution STM image, in which phase-3 molecular arrays are regularly distributed with the nanopores with size of $\sim 2.0 \mathrm{~nm}$ in diameter. A highresolution STM image in Figure 3e shows that six 3Br-PTM molecules assembled into a hexagonal nanopore. DFT-optimized molecular packing structure in Figure $3 \mathrm{f}$ suggests that each periphery of the hexagonal nanopore consists of two 3Br-PTM molecules that aligns via linear single Br-bond (Type-1 halogen bond, as discussed in Figure 4a). Figure S7 displays mixed phase-3 and phase-1 of 3Br-PTM on Br-monolayer on Au(111). Phase-3 mostly formed small domain, and its structural instability affects the STM-tip state during scanning. 

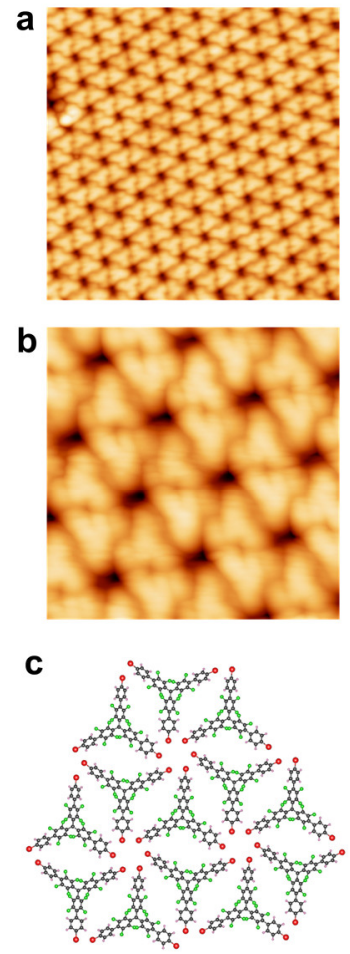

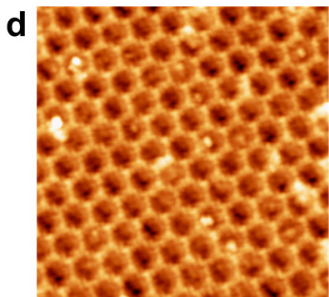

e
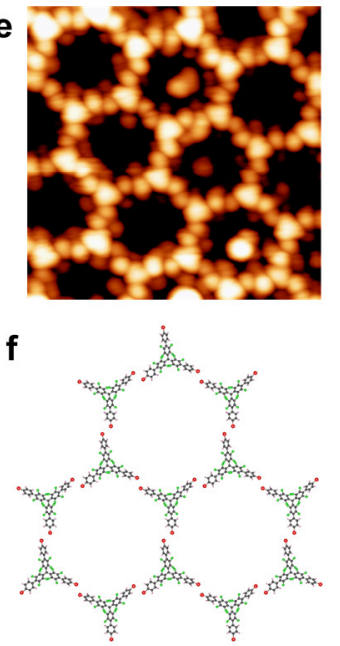
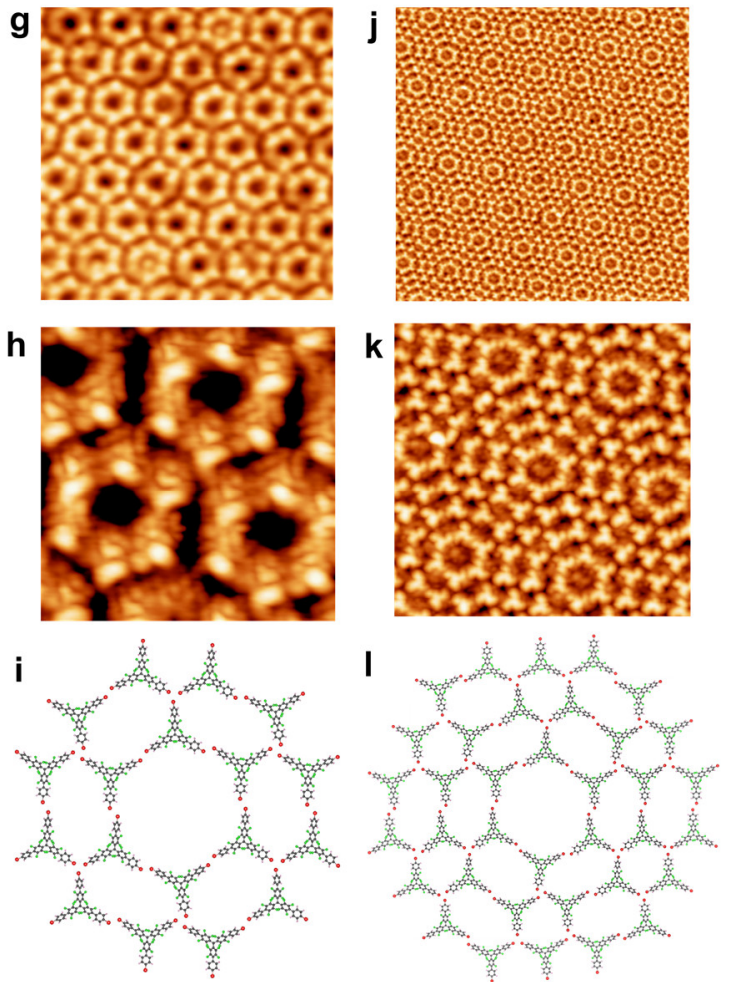

Figure 3. Multi-phases of 3Br-PTM self-assembled on Br-monolayer on $\mathrm{Au}(111)$. (a, b, c) STM images and DFT-optimized molecular packing structure of phase-2 at high coverage at room temperature; set point: a) $V_{\text {tip }}=-0.2 \mathrm{~V}, I_{\text {tip }}=25 \mathrm{pA}$, and size: $25 \mathrm{~nm} \times 25 \mathrm{~nm}$; b) $V_{\text {tip }}=-$ $0.5 \mathrm{~V}, I_{\text {tip }}=25 \mathrm{pA}$, and size: $8 \mathrm{~nm} \times 8 \mathrm{~nm}$. (d, e, f) STM images and DFT-optimized molecular packing structure of phase-3 at high coverage; set point: d) $V_{\text {tip }}=0.8 \mathrm{~V}, I_{\text {tip }}=30 \mathrm{pA}$, and size: $25 \mathrm{~nm} \times 25 \mathrm{~nm}$; e) $V_{t i p}=0.5 \mathrm{~V}, I_{\text {tip }}=21 \mathrm{pA}$, and size: $8 \mathrm{~nm} \times 8 \mathrm{~nm}$. $(\mathrm{g}, \mathrm{h}$, i) STM images and DFT-optimized molecular packing structure of phase-4 at high coverage; set point: g) $V_{\text {tip }}=1.2 \mathrm{~V}, I_{\text {tip }}=54 \mathrm{pA}$, and size: $25 \mathrm{~nm} \times 25 \mathrm{~nm}$; h) $V_{\text {tip }}=-1.5 \mathrm{~V}, I_{\text {tip }}=130 \mathrm{pA}$, and size: $8 \mathrm{~nm} \times 8 \mathrm{~nm}$. (j, k, 1) STM images and proposed molecular packing structure of phase-5 observed after annealing at $250{ }^{\circ} \mathrm{C}$; set point: j) $V_{\text {tip }}=0.8 \mathrm{~V}, I_{\text {tip }}=29 \mathrm{pA}$, and size: $50 \mathrm{~nm} \times 50$ $\mathrm{nm}$; k) $V_{\text {tip }}=0.8 \mathrm{~V}, I_{\text {tip }}=29 \mathrm{pA}$, and size: $18 \mathrm{~nm} \times 18 \mathrm{~nm}$.

Like Phase-3, Phase-4 also has a regular hexagonal porous structure. Figure $3 \mathrm{~g}$ gives a STM image of phase- 4 molecular array with regularly distributed but isolated nanopores. The zoom-in STM image in Figure $3 \mathrm{~h}$ shows that six 3Br-PTM molecules assemble into a hexagonal nanopore. Unlike phase-3, the porous walls (molecules) in phase-4 are independent and do not share with other nanopores. DFT-optimized molecular packing structure in Figure $3 \mathrm{i}$ verifies our finding, suggesting that the wall comprises of two $3 \mathrm{Br}-\mathrm{PTM}$ molecules that 
align via a linear single Br-bond (Type-1 halogen bond, same as phase-3). Further, DFToptimized structure suggests the formation of triangular triple Br-bonds (Type-2 halogen bond, as observed in phase-1), which bridges the isolated nanopores into 2D porous molecular spin arrays. Figure S8 exhibits a large-scale STM image of phase-4 of 3Br-PTM on Br-monolayer on $\mathrm{Au}(111)$. The formation of high yield of ordered nanostructures indicates that phase-4 has a good structure stability.

Phases 1-4 are mixed at high molecular coverage before annealing. Statistically, the statistical yields of phase $1-4$ are $42 \%, 29 \%, 5 \%$ and $24 \%$, respectively. Table S1 summarizes the observed molecular phases at different annealing temperatures. According to Table S1, Phase 1, 2 and 4 remain stable at below $200{ }^{\circ} \mathrm{C}$. We infer that phase- 3 is unstable since it disappears when annealing at $200{ }^{\circ} \mathrm{C}$. Due to its low stability, the statistical yield of phase-3 is only $\sim 5 \%$ at room temperature.

Unexpectedly, a new phase- 5 emerged upon the sample annealed at $250{ }^{\circ} \mathrm{C}$. Figure $3 \mathrm{j}$ presents the phase-5 molecular array that resembles $\mathrm{Si}(7 \times 7)$ reconstruction-like structure. Figure $\mathrm{S} 9$ gives large-scale STM images of mixed phase-5 and phase-1 of 3Br-PTM on Br-monolayer on $\mathrm{Au}(111)$. A high-resolution STM image in Figure 3k and DFT-optimized molecular packing structure in Figure $3 \mathrm{i}$ suggest that $3 \mathrm{Br}-\mathrm{PTM}$ molecules assemble into $\mathrm{Si}(7 \times 7)$ reconstruction-like arrays, formed by the combination of two types of halogen bonds, namely the linear single Br-bond (Type-1 halogen bond) and triangular triple Br-bond (Type-2 halogen bond). Although the supramolecular arrangements are somewhat different, phase-5 and phase-4 are similar in terms of the molecular interactions involved. We note that the $\mathrm{Si}(7 \times 7)$ reconstruction-like spin array is obtained in large scale of $200 \mathrm{~nm} \times 200 \mathrm{~nm}$ (Figure S10), indicating that phase-5 is energetically stable.

We infer that the formation of the above multi-phases is mainly governed from their diverse intermolecular interactions, while the molecule-substrate interaction does not play an important role in determining the self-assembled networks. The Br-monolayer is considered 
as an insulating layer ${ }^{54}$ that is electronically inactive, and therefore it can be used for an ultrathin buffer layer for molecular-based devices. DFT calculation also verifies that the molecule-substrate interaction of 3Br-PTM on Br-monolayer is weak ( $\sim 81 \mathrm{meV}$ per atom). To understand the inherent mechanism of the formation of the five observed phases, further DFT calculations were performed in order to gain insight of respective intermolecular interactions.

a

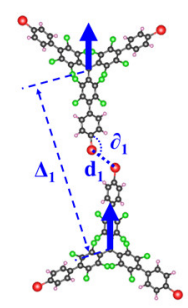

Type 1: linear single Br-bond Type-2: triangular triple Br-bonds Bond distance: $\mathrm{d}_{1}=3.8 \pm 0.2 \AA$ Bond angle: $\partial_{1}=118 \pm 10^{\circ}$ Spin distance: $\Delta_{1}=23.1 \pm 0.3 \AA$

d

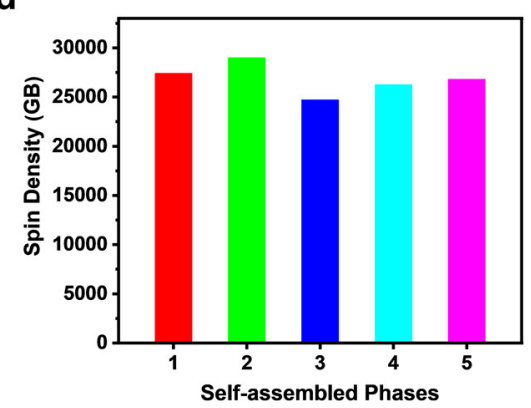

b

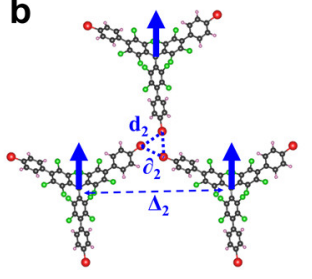

Bond distance: $d_{2}=3.6 \pm 0.1$ Bond angle: $\partial_{2}=60 \pm 2^{\circ}$ Bond angle: $\partial_{2}=60 \pm 2$
Spin distance: $\Delta_{2}=21.3 \pm 0.1 \AA$ c

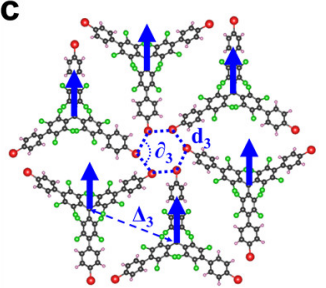

Type-3: hexagonal sextuple Br-bonds Bond distance: $\mathrm{d}_{3}=3.6 \pm 0.2 \AA$ Bond angle: $\partial_{3}=119 \pm 6^{\circ}$ Spin distance: $\Delta_{3}=13.6 \pm 0.2 \AA$

e

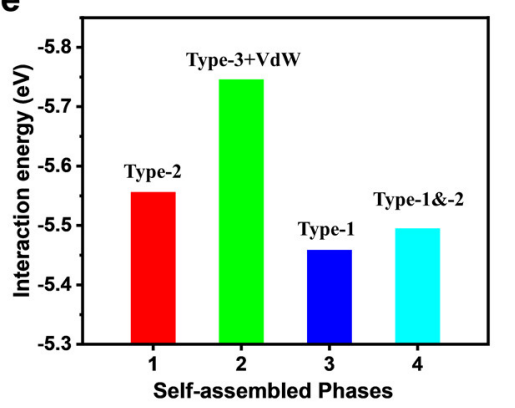

Figure 4. The formation of five different phases stabled by the diverse Br-halogen bonds. (a, b, c) DFT-optimized model of three types of Br-halogen bonds: Type-1 halogen bond: linear single Br-bond, Type-2 halogen bond: triangular triple Br-bonds and Type-3 halogen bond: hexagonal sextuple Br-bonds. (d) Spin density at the size of a flash drive in phase 1-5. (e) The corresponding intermolecular interactions energy per molecule in Phase 1-4, the dominant intermolecular interactions are added.

We studied the three types of molecular interaction that are involved in the five phases, namely, type-1 halogen bond: linear single Br-bond, type-2 halogen bond: triangular triple $\mathrm{Br}-$ bonds and type-3 halogen bond: hexagonal sextuple Br-bonds, respectively. Figure $4 a-4 c$ are three corresponding optimized molecular models after formation energy minimisation. Halogen bond forms based on the mutual interaction between $\delta$-hole and electron-rich 
equatorial belt due to the anisotropic distribution of halogen atom's electron density. ${ }^{55,56}$ Due to this distribution, the electron density rearrangement leads to the coexistence of electrondepleted $\delta$-hole at the pole of the $\mathrm{C}-\mathrm{X}$ bond ( $\mathrm{X}$ indicates halogen atom), and encircling electron-rich equatorial belt arises perpendicular to the $\mathrm{C}-\mathrm{X}$ bond. Figure $4 \mathrm{a}$ shows the type-1 halogen bond where linear single Br-bond exists. In this scenario, two 3Br-PTM molecules are aligned such that two $\mathrm{Br}$ atoms are in close proximity. The $\mathrm{Br}-\mathrm{Br}$ distance $\left(\mathrm{d}_{1}\right)$ is calculated to be about $\sim 3.8 \AA$, considered to be within the range of short intermolecular interactions, suggesting the formation of halogen bonding interactions. In addition, we found that the bond angle $\left(\partial_{1}\right)$ is $\sim 118^{\circ}$. We note that our result is in line to the type- 1 halogen bond that was theoretically predicted in 2017.55 The probability of phase-3's statistical yield is very low (only 5\%), and this is probably because of the small binding energy of involved type-1 halogen bond that impedes such an arrangement.

In contrast, type- 2 halogen bond of triangular triple Br-bonds has been frequently reported. ${ }^{46}$, ${ }^{55}$ Figure $4 \mathrm{~b}$ shows that three $\mathrm{Br}$ atoms positions are adjacent to each other, and they form a trigonal geometry after structural optimization. The bond angle $\left(\partial_{2}\right)$ and $\operatorname{Br}-\mathrm{Br}$ distance $\left(\mathrm{d}_{2}\right)$ are $\sim 60^{\circ}$ and $\sim 3.6 \AA$, respectively. These geometrical parameters are fairly comparable to the reported case. ${ }^{55}$ Type- 3 halogen bond of hexagonal sextuple Br-bonds is resolved in phase-2. DFT-optimized model in Figure $4 \mathrm{c}$ shows that six $\mathrm{Br}$ atoms form a hexagonal-shaped structure with the bond angle $\left(\partial_{3}\right)$ of $\sim 119^{\circ}$ and $\mathrm{Br}-\mathrm{Br}$ distance $\left(\mathrm{d}_{3}\right)$ of $\sim 3.6 \AA$. Type- 3 halogen bond has been theoretically predicted, ${ }^{55}$ and it was recently demonstrated by Maier et al. in experiment. ${ }^{45}$ We noted that the types of halogen bonds are diverse, which can be employed to realize variable molecular self-assembled structures and thus adjusting the lateral distance of two molecular spins. Here we recorded three different distances between two molecular spins: 13.6 ̊, $21.3 \AA$ and $23.1 \AA$, respectively. Moreover, different molecular interactions or spin distances leads to variable spin densities. Figure $4 \mathrm{~d}$ summarizes the molecular spin density throughout the five phases. The maximum spin density with the value of $3 \times 10^{13}$ 
spins (29000 GB) at the size of a flash drive is found in highly-packed phase-2 (The fifth row at Table 1), which is greatly larger than the $256 \mathrm{~GB}$ data storage of a commercial flash drive. Intermolecular interaction energy is also extracted from DFT calculations shown in Figure 4e. Phase-3 has a minimum intermolecular interaction energy compared to the other phases, which further confirms our experimental observation that its structure is unstable and its statistical yield is lowest. On the contrary, the intermolecular interaction energy of phase-1 is larger than that of phase-3, suggesting that phase- 1 is more stable than phase-3. According to the intermolecular interaction energy, we infer an order of structural stability: phase-1>phase$4>$ phase-3, which is consist with our experimental observation of the statistical yield order of phase- $1>$ phase- $4>$ phase- 3 .

Table 1. Summary of the calculated interaction energy, the bond types of molecular interaction, the molecular density and the spin density in Phase 1-5.

\begin{tabular}{llllll}
\hline Items & Phase- 1 & Phase-2 & Phase-3 & Phase- 4 & Phase-5 \\
\hline $\begin{array}{l}\text { Interaction energy } \\
(\mathrm{eV})^{[\mathrm{a}]}\end{array}$ & -5.56 & -5.75 & -5.46 & -5.50 & -- \\
\hline $\begin{array}{l}\text { Type of molecular } \\
\text { interaction }\end{array}$ & Type-2 & $\begin{array}{l}\text { Type-3 } \\
\& \text { vdW }\end{array}$ & Type-1 & $\begin{array}{l}\text { Type-1 } \\
\text { \&type-2 }\end{array}$ & $\begin{array}{l}\text { Type-1 } \\
\text { \&type-2 }\end{array}$ \\
\hline $\begin{array}{l}\text { Molecular density } \\
\left(\text { molecule/103 } \mathrm{nm}^{2}\right)\end{array}$ & 409 & 433 & 370 & 392 & 400 \\
\hline $\begin{array}{l}\text { Spin density } \\
(\mathrm{GB})^{[\mathrm{b}]}\end{array}$ & 27400 & 29000 & 24790 & 26260 & 26800 \\
\hline
\end{tabular}

[a] interaction energy per molecule, [b] at the size of a flash drive

The intermolecular interaction energy of phase- 2 is the highest than the other phases. We attributed this to two origins, type-3 halogen bond and the van der Waals force (vdW). The vdW force also plays an important role in Phase-2 due to its highly-packed molecular density. The fourth row at Table 1 shows that phase- 2 has the highest molecular density, and STM image in Figure $3 \mathrm{~b}$ shows that the 3Br-PTM molecules are closely packed. Phase- 4 has a 
moderate intermolecular interaction energy than that of phase-1 and phase-3 (Table 1 and Figure 4e), which is attributed to a combination of type- 1 and type- 2 halogen bonds. We cannot obtain the optimized structure of phase-5, since its unit cell is very large and has exceeded our available computing capacity. Nevertheless, the molecular interaction energy of phase- 5 is expected to fall in the range of phase- 1 and phase- 3 , since phase- 5 is arranged from a combination of type- 1 and type- 2 halogen bonds, same as the case of phase- 4 .

Based on the DFT calculations and our experimental observations, we infer that the formation of five phases is mainly driven by different formation of halogen bonds, and the dominant types of molecular interaction are added in Figure 4e. We note that the formation of halogen bond/phases depends on molecular density and annealing temperature. Molecular coverage and annealing treatment are thus utilized to adjust the molecular spin arrays to some extent. At low coverage, we only observed the existence of phase-1, which may be related to the dominant interaction of type- 2 halogen bond. In the other hand, at high coverage, phase- 2 is commonly found due to its highest molecular density (fourth row of Table 1) that lead to the second dominant interaction of $\mathrm{vdW}$ force, in addition to the type-2 halogen bond. Whereas, the other observed phases form locally, which may be because 3Br-PTM molecules are not uniformly distributed on surface. Table S1 summarizes that effect of annealing treatment on different self-assembled phases. Annealing to $200{ }^{\circ} \mathrm{C}$, phase- 3 disappears since the involved type-1 halogen bond is weak, while phase- $1,-2 \&-4$ remain. The emergence of phase- 5 with a long-scale order of $200 \mathrm{~nm} \times 200 \mathrm{~nm}$ is observed when the sample was annealed at $250{ }^{\circ} \mathrm{C}$.

\section{Conclusion}

In conclusion, two-dimensional supramolecular spin arrays were realized on an insulating $\mathrm{Br}$ monolayer on $\mathrm{Au}(111)$. In this study, we found that the halogen bond rather than moleculesubstrate interaction is the main determinant of the supramolecular spin arrays formation. By taking advantage of the diversity of halogen bonds, five kinds of two-dimensional supramolecular architectures were achieved with variable spin densities and spin distances. 
DFT calculations support our STM observations, suggesting that the formation of three types of halogen bonds realizes the $2 \mathrm{D}$ spin arrays. Our work demonstrates how versatile the halogen bond on the verge of $2 \mathrm{D}$ spin arrays realization on a surface, suggesting that supramolecular self-assembly facilitated by halogen bonding as a promising method to engineer low-dimensional spin arrays towards next-generation magnetic device applications. Further work is needed to read/write each individual molecular spin and to precisely engineer the supramolecular self-assembly of single-molecule magnets and tune effectively their spin exchange. $^{57}$

\section{Acknowledgements}

We acknowledge financial support from MOE Tier 2 projects (MOE2018-T2-1-088 and MOE2018-T2-2-094), NRF-NSFC grant R-144-000-405-281, and A*STAR AME YIRG project (A2084c0164). The authors would like to acknowledge the Singapore Synchrotron Light Source (SSLS) for providing the facility necessary for conducting the research. The SSLS is a National Research Infrastructure under the National Research Foundation Singapore. We also acknowledge Dr. Xiaojiang Yu from SSLS for technical support. We would like to thank the Centre for Advanced 2D materials and Center of Information Technology at NUS for the computational resource.

\section{References}

(1) Goodwin, C. A. P.; Ortu, F.; Reta, D.; Chilton, N. F.; Mills, D. P., Molecular Magnetic Hysteresis at 60 Kelvin in Dysprosocenium. Nature 2017, 548, 439-442.

(2) Guo, F.-S.; Day, B. M.; Chen, Y.-C.; Tong, M.-L.; Mansikkamäki, A.; Layfield, R. A., A Dysprosium Metallocene Single-Molecule Magnet Functioning at the Axial Limit. Angew. Chem. Int. Ed. 2017, 56, 11445-11449.

(3) Gaita-Ariño, A.; Luis, F.; Hill, S.; Coronado, E., Molecular Spins for Quantum Computation. Nat. Chem. 2019, 11, 301-309.

(4) Leuenberger, M. N.; Loss, D., Quantum Computing in Molecular Magnets. Nature 2001, 410, 789-793.

(5) Krainov, I. V.; Klier, J.; Dmitriev, A. P.; Klyatskaya, S.; Ruben, M.; Wernsdorfer, W.; Gornyi, I. V., Giant Magnetoresistance in Carbon Nanotubes with Single-Molecule Magnets Tbpc2. ACS Nano 2017, 11, 6868-6880.

(6) Pinkowicz, D.; Chorąży, S.; Stefańczyk, O., An Invitation to Molecular Magnetism. Science Progress 2011, 94, 139-183. 
(7) Sessoli, R.; Tsai, H. L.; Schake, A. R.; Wang, S.; Vincent, J. B.; Folting, K.; Gatteschi, D.; Christou, G.; Hendrickson, D. N., High-Spin Molecules: [Mn12o12(O2cr)16(H2o)4]. J. Am. Chem. Soc. 1993, 115, 1804-1816.

(8) Day, B. M.; Guo, F.-S.; Layfield, R. A., Cyclopentadienyl Ligands in Lanthanide SingleMolecule Magnets: One Ring to Rule Them All? Acc. Chem. Res. 2018, 51, 1880-1889.

(9) Liu, J.-L.; Chen, Y.-C.; Tong, M.-L., Symmetry Strategies for High Performance Lanthanide-Based Single-Molecule Magnets. Chem. Soc. Rev. 2018, 47, 2431-2453.

(10) Marin, R.; Brunet, G.; Murugesu, M., Shining New Light on Multifunctional Lanthanide Single-Molecule Magnets. Angew. Chem. Int. Ed. 2021, 60, 1728-1746.

(11) Yu, K.-X.; Kragskow, J. G. C.; Ding, Y.-S.; Zhai, Y.-Q.; Reta, D.; Chilton, N. F.; Zheng, Y.-Z., Enhancing Magnetic Hysteresis in Single-Molecule Magnets by Ligand Functionalization. Chem 2020, 6, 1777-1793.

(12) Jin, P.-B.; Zhai, Y.-Q.; Yu, K.-X.; Winpenny, R. E. P.; Zheng, Y.-Z., Dysprosiacarboranes as Organometallic Single-Molecule Magnets. Angew. Chem. Int. Ed. 2020, 59, 9350-9354.

(13) Y. Gopalakrishna, T.; Zeng, W.; Lu, X.; Wu, J., From Open-Shell Singlet Diradicaloids to Polyradicaloids. Chemi. Commun. 2018, 54, 2186-2199.

(14) Liu, C.; Sandoval-Salinas, M. E.; Hong, Y.; Gopalakrishna, T. Y.; Phan, H.; Aratani, N.; Herng, T. S.; Ding, J.; Yamada, H.; Kim, D.; Casanova, D.; Wu, J., Macrocyclic Polyradicaloids with Unusual Super-Ring Structure and Global Aromaticity. Chem 2018, 4, 1586-1595.

(15) Katoh, K.; Yamashita, S.; Yasuda, N.; Kitagawa, Y.; Breedlove, B. K.; Nakazawa, Y.; Yamashita, M., Control of the Spin Dynamics of Single-Molecule Magnets by Using a Quasi One-Dimensional Arrangement. Angew. Chem. Int. Ed. 2018, 57, 9262-9267.

(16) Liu, J.; Ravat, P.; Wagner, M.; Baumgarten, M.; Feng, X.; Müllen, K., Tetrabenzo[a,F,J,O]Perylene: A Polycyclic Aromatic Hydrocarbon with an Open-Shell Singlet Biradical Ground State. Angew. Chem. Int. Ed. 2015, 54, 12442-12446.

(17) Zeng, W.; Sun, Z.; Herng, T. S.; Gonçalves, T. P.; Gopalakrishna, T. Y.; Huang, K.-W.; Ding, J.; Wu, J., Super-Heptazethrene. Angew. Chem. Int. Ed. 2016, 55, 8615-8619.

(18) Aulakh, D.; Liu, L.; Varghese, J. R.; Xie, H.; Islamoglu, T.; Duell, K.; Kung, C.-W.; Hsiung, C.-E.; Zhang, Y.; Drout, R. J.; Farha, O. K.; Dunbar, K. R.; Han, Y.; Wriedt, M., Direct Imaging of Isolated Single-Molecule Magnets in Metal-Organic Frameworks. J. Am. Chem. Soc. 2019, 141, 2997-3005.

(19) Laskowska, M.; Pastukh, O.; Kuźma, D.; Laskowski, Ł., How to Control the Distribution of Anchored, Mn12-Stearate, Single-Molecule Magnets. Nanomaterials 2019, 9, 1730.

(20) Gómez-Segura, J.; Veciana, J.; Ruiz-Molina, D., Advances on the Nanostructuration of Magnetic Molecules on Surfaces: The Case of Single-Molecule Magnets (Smm). Chemi. Commun. 2007, 3699-3707.

(21) Köbke, A.; Gutzeit, F.; Röhricht, F.; Schlimm, A.; Grunwald, J.; Tuczek, F.; Studniarek, M.; Longo, D.; Choueikani, F.; Otero, E.; Ohresser, P.; Rohlf, S.; Johannsen, S.; Diekmann, F.; Rossnagel, K.; Weismann, A.; Jasper-Toennies, T.; Näther, C.; Herges, R.; Berndt, R.; Gruber, M., Reversible Coordination-Induced Spin-State Switching in Complexes on Metal Surfaces. Nat. Nanotech. 2020, 15, 18-21.

(22) Serrano, G.; Poggini, L.; Briganti, M.; Sorrentino, A. L.; Cucinotta, G.; Malavolti, L.; Cortigiani, B.; Otero, E.; Sainctavit, P.; Loth, S.; Parenti, F.; Barra, A.-L.; Vindigni, A.; Cornia, A.; Totti, F.; Mannini, M.; Sessoli, R., Quantum Dynamics of a Single Molecule Magnet on Superconducting $\mathrm{Pb}(111)$. Nat. Mater. 2020, 19, 546-551.

(23) Cini, A.; Mannini, M.; Totti, F.; Fittipaldi, M.; Spina, G.; Chumakov, A.; Rüffer, R.; Cornia, A.; Sessoli, R., Mössbauer Spectroscopy of a Monolayer of Single Molecule Magnets. Nat. Commun. 2018, 9, 480. 
(24) Mishra, S.; Lohr, T. G.; Pignedoli, C. A.; Liu, J.; Berger, R.; Urgel, J. I.; Müllen, K.; Feng, X.; Ruffieux, P.; Fasel, R., Tailoring Bond Topologies in Open-Shell Graphene Nanostructures. ACS Nano 2018, 12, 11917-11927.

(25) Liu, J.; Mishra, S.; Pignedoli, C. A.; Passerone, D.; Urgel, J. I.; Fabrizio, A.; Lohr, T. G.; Ma, J.; Komber, H.; Baumgarten, M.; Corminboeuf, C.; Berger, R.; Ruffieux, P.; Müllen, K.; Fasel, R.; Feng, X., Open-Shell Nonbenzenoid Nanographenes Containing Two Pairs of Pentagonal and Heptagonal Rings. J. Am. Chem. Soc. 2019, 141, 12011-12020.

(26) Su, J.; Telychko, M.; Hu, P.; Macam, G.; Mutombo, P.; Zhang, H.; Bao, Y.; Cheng, F.; Huang, Z.-Q.; Qiu, Z.; Tan, S. J. R.; Lin, H.; Jelínek, P.; Chuang, F.-C.; Wu, J.; Lu, J., Atomically Precise Bottom-up Synthesis of ח-Extended [5]Triangulene. Sci. Adv. 2019, 5, eaav7717.

(27) Li, J.; Sanz, S.; Castro-Esteban, J.; Vilas-Varela, M.; Friedrich, N.; Frederiksen, T.; Peña, D.; Pascual, J. I., Uncovering the Triplet Ground State of Triangular Graphene Nanoflakes Engineered with Atomic Precision on a Metal Surface. Phys. Rev. Lett. 2020, 124, 177201.

(28) Pavliček, N.; Mistry, A.; Majzik, Z.; Moll, N.; Meyer, G.; Fox, D. J.; Gross, L., Synthesis and Characterization of Triangulene. Nat. Nanotech. 2017, 12, 308-311.

(29) Gragnaniello, L.; Paschke, F.; Erler, P.; Schmitt, P.; Barth, N.; Simon, S.; Brune, H.; Rusponi, S.; Fonin, M., Uniaxial 2d Superlattice of Fe4 Molecular Magnets on Graphene. Nano Lett. 2017, 17, 7177-7182.

(30) Paschke, F.; Erler, P.; Enenkel, V.; Gragnaniello, L.; Fonin, M., Bulk-Like Magnetic Signature of Individual Fe4h Molecular Magnets on Graphene. ACS Nano 2019, 13, 780-785.

(31) Goronzy, D. P.; Ebrahimi, M.; Rosei, F.; Arramel; Fang, Y.; De Feyter, S.; Tait, S. L.; Wang, C.; Beton, P. H.; Wee, A. T. S.; Weiss, P. S.; Perepichka, D. F., Supramolecular Assemblies on Surfaces: Nanopatterning, Functionality, and Reactivity. ACS Nano 2018, 12, $7445-7481$.

(32) Huang, Y. L.; Chen, W.; Li, H.; Ma, J.; Pflaum, J.; Wee, A. T. S., Tunable TwoDimensional Binary Molecular Networks. Small 2010, 6, 70-75.

(33) Barth, J. V., Molecular Architectonic on Metal Surfaces. Annu. Rev. Phys. Chem. 2007, $58,375-407$.

(34) Otero, R.; Gallego, J. M.; de Parga, A. L. V.; Martín, N.; Miranda, R., Molecular SelfAssembly at Solid Surfaces. Adv. Mater. 2011, 23, 5148-5176.

(35) Goiri, E.; Borghetti, P.; El-Sayed, A.; Ortega, J. E.; de Oteyza, D. G., Multi-Component Organic Layers on Metal Substrates. Adv. Mater. 2016, 28, 1340-1368.

(36) Wasio, N. A.; Quardokus, R. C.; Forrest, R. P.; Lent, C. S.; Corcelli, S. A.; Christie, J. A.; Henderson, K. W.; Kandel, S. A., Self-Assembly of Hydrogen-Bonded Two-Dimensional Quasicrystals. Nature 2014, 507, 86-89.

(37) Cheng, F.; Wu, X.-J.; Hu, Z.; Lu, X.; Ding, Z.; Shao, Y.; Xu, H.; Ji, W.; Wu, J.; Loh, K. P., Two-Dimensional Tessellation by Molecular Tiles Constructed from Halogen-Halogen and Halogen-Metal Networks. Nat. Commun. 2018, 9, 4871.

(38) Cai, L.; Sun, Q.; Bao, M.; Ma, H.; Yuan, C.; Xu, W., Competition between Hydrogen Bonds and Coordination Bonds Steered by the Surface Molecular Coverage. ACS Nano 2017, 11, 3727-3732.

(39) Lin, X.; Lu, J. C.; Shao, Y.; Zhang, Y. Y.; Wu, X.; Pan, J. B.; Gao, L.; Zhu, S. Y.; Qian, K.; Zhang, Y. F.; Bao, D. L.; Li, L. F.; Wang, Y. Q.; Liu, Z. L.; Sun, J. T.; Lei, T.; Liu, C.; Wang, J. O.; Ibrahim, K.; Leonard, D. N.; Zhou, W.; Guo, H. M.; Wang, Y. L.; Du, S. X.; Pantelides, S. T.; Gao, H. J., Intrinsically Patterned Two-Dimensional Materials for Selective Adsorption of Molecules And nanoclusters. Nat. Mater. 2017, 16, 717-721.

(40) Wang, Q. H.; Hersam, M. C., Room-Temperature Molecular-Resolution Characterization of Self-Assembled Organic Monolayers on Epitaxial Graphene. Nat. Chem. 2009, 1, 206-211.

(41) Stepanow, S.; Lingenfelder, M.; Dmitriev, A.; Spillmann, H.; Delvigne, E.; Lin, N.; Deng, X.; Cai, C.; Barth, J. V.; Kern, K., Steering Molecular Organization and Host-Guest 
Interactions Using Two-Dimensional Nanoporous Coordination Systems. Nat. Mater. 2004, 3, 229-233.

(42) Lin, T.; Shang, X. S.; Adisoejoso, J.; Liu, P. N.; Lin, N., Steering on-Surface Polymerization with Metal-Directed Template. J. Am. Chem. Soc. 2013, 135, 3576-3582.

(43) Xing, L.; Jiang, W.; Huang, Z.; Liu, J.; Song, H.; Zhao, W.; Dai, J.; Zhu, H.; Wang, Z.; Weiss, P. S.; Wu, K., Steering Two-Dimensional Porous Networks with $\Sigma$-Hole Interactions of $\mathrm{Br} \cdots \mathrm{S}$ and $\mathrm{Br} \cdots \mathrm{Br}$. Chem. Mater. 2019, 31, 3041-3048.

(44) Shang, J.; Wang, Y.; Chen, M.; Dai, J.; Zhou, X.; Kuttner, J.; Hilt, G.; Shao, X.; Gottfried, J. M.; Wu, K., Assembling Molecular Sierpiński Triangle Fractals. Nat. Chem. 2015, 7, 389-393.

(45) Yang, Z.; Fromm, L.; Sander, T.; Gebhardt, J.; Schaub, T. A.; Görling, A.; Kivala, M.; Maier, S., On-Surface Assembly of Hydrogen- and Halogen-Bonded Supramolecular Graphyne-Like Networks. Angew. Chem. Int. Ed. 2020, 59, 9549-9555.

(46) Teyssandier, J.; Mali, K. S.; De Feyter, S., Halogen Bonding in Two-Dimensional Crystal Engineering. ChemistryOpen 2020, 9, 225-241.

(47) Wu, S.; Li, M.; Phan, H.; Wang, D.; Herng, T. S.; Ding, J.; Lu, Z.; Wu, J., Toward TwoDimensional П-Conjugated Covalent Organic Radical Frameworks. Angew. Chem. Int. Ed. 2018, 57, 8007-8011.

(48) Smykalla, L.; Shukrynau, P.; Korb, M.; Lang, H.; Hietschold, M., Surface-Confined 2d Polymerization of a Brominated Copper-Tetraphenylporphyrin on $\mathrm{Au}(111)$. Nanoscale 2015, 7, 4234-4241.

(49) Wang, D.; Yang, M.; Arramel; Wu, J.; Wee, A. T. S., Thermally Induced Chiral Aggregation of Dihydrobenzopyrenone on Au(111). ACS Appl. Mater. Interfaces 2020, 12, 35547-35554.

(50) Huang, Z.; Lin, Y.; Han, C.; Sun, Y.-Y.; Wu, K.; Chen, W., Atomic-Scale Local Work Function Characterizations of $\mathrm{Br}$ Islands on $\mathrm{Cu}(111)$. J. Phys. Chem. C 2021, 125, 7944-7949. (51) Rizzo, D. J.; Veber, G.; Cao, T.; Bronner, C.; Chen, T.; Zhao, F.; Rodriguez, H.; Louie, S. G.; Crommie, M. F.; Fischer, F. R., Topological Band Engineering of Graphene Nanoribbons. Nature 2018, 560, 204-208.

(52) Tsai, H.-Z.; Omrani, A. A.; Coh, S.; Oh, H.; Wickenburg, S.; Son, Y.-W.; Wong, D.; Riss, A.; Jung, H. S.; Nguyen, G. D.; Rodgers, G. F.; Aikawa, A. S.; Taniguchi, T.; Watanabe, K.; Zettl, A.; Louie, S. G.; Lu, J.; Cohen, M. L.; Crommie, M. F., Molecular Self-Assembly in a Poorly Screened Environment: F4tenq on Graphene/Bn. ACS Nano 2015, 9, 12168-12173.

(53) Schmid, M.; Kaftan, A.; Steinrück, H.-P.; Gottfried, J. M., The Electronic Structure of Cobalt(Ii) Phthalocyanine Adsorbed on Ag(111). Surf. Sci. 2012, 606, 945-949.

(54) Drost, R.; Ojanen, T.; Harju, A.; Liljeroth, P., Topological States in Engineered Atomic Lattices. Nat. Phys. 2017, 13, 668-671.

(55) Han, Z.; Czap, G.; Chiang, C.-1.; Xu, C.; Wagner, P. J.; Wei, X.; Zhang, Y.; Wu, R.; Ho, W., Imaging the Halogen Bond in Self-Assembled Halogenbenzenes on Silver. Science 2017, 358, 206-210.

(56) Wang, D.; Wang, Z.; Liu, W.; Arramel; Zhou, J.; Feng, Y. P.; Loh, K. P.; Wu, J.; Wee, A. T. S., Atomic-Level Electronic Properties of Carbon Nitride Monolayers. ACS Nano 2020, 14, 14008-14016.

(57) Li Huang, Y.; Lu, Y.; Niu, T. C.; Huang, H.; Kera, S.; Ueno, N.; Wee, A. T. S.; Chen, W., Reversible Single-Molecule Switching in an Ordered Monolayer Molecular Dipole Array. Small 2012, 8, 1423-1428. 


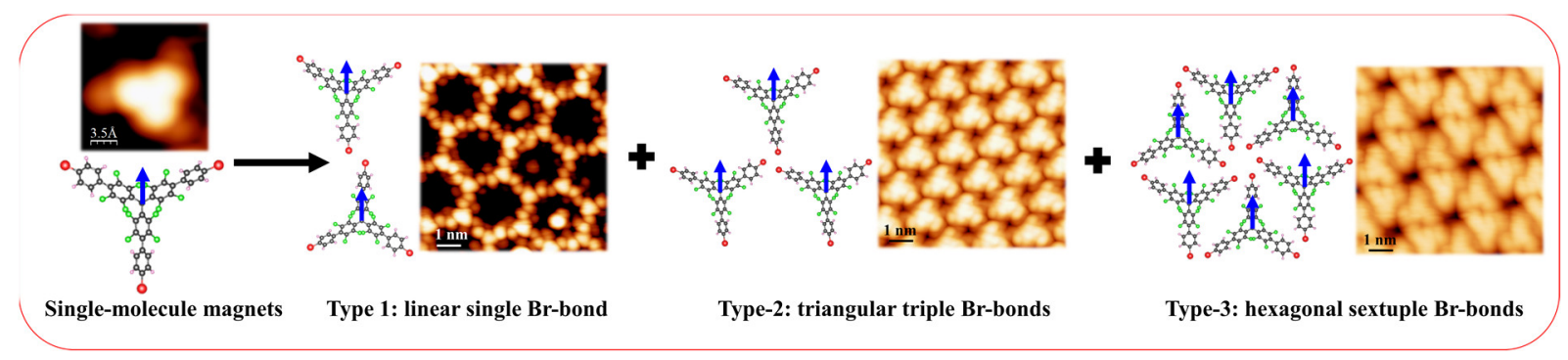

TOC. A bromine-terminal perchlorotriphenylmethyl radical was synthesized and deposited on $\mathrm{Au}(111)$ to realize 2D spin arrays. By taking advantage of the diversity of halogen bonds, several supramolecular spin arrays were achieved with variable spin densities/distances. DFT calculation suggests that the STM-observed spin arrays are governed by three types of halogen bonds. 